\title{
Cambios, (des)igualdades y día a día del profesional de la abogacía
}

\author{
Nuria Codina \\ José Vicente Pestana \\ Universidad de Barcelona. Departamento de Psicología Social \\ ncodina@ub.edu; jvpestana@ub.edu
}

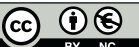

Recibido: 09-09-2011

Aceptado: 26-09-2012

\section{Resumen}

La raigambre y el peso de la abogacía en la sociedad española confieren un interés especial al conocimiento sobre su estado actual y a los cambios observados en sus profesionales. El objetivo de esta nota de investigación es poner de manifiesto los indicadores de desprofesionalización y (des)igualdades de género en el ejercicio del derecho. Este propósito se concretó mediante entrevistas realizadas a una muestra de 562 profesionales ejercitantes (335 abogados y 227 abogadas). Específicamente, se describen y se valoran los perfiles demográfico (sexo, edad), personal (núcleo familiar y relaciones con la abogacía), de formación y ejercicio profesional (educación previa y licenciatura posterior, inicios en la profesión y ejercicio actual de la misma). Los análisis practicados constatan, en la generación más joven de abogadas y abogados, una mayoría de mujeres y una menor presencia de antecedentes familiares en el mundo del derecho. La desprofesionalización de la abogacía se pone de manifiesto en las diferencias entre jóvenes y no jóvenes con respecto a los inicios en el ejercicio de la profesión y su posición actual en el contexto del despacho profesional. Entre abogados y abogadas, se advierten (des)igualdades en la formación y el ejercicio actual de las ramas del derecho, así como en las horas trabajadas a lo largo de la semana. Los datos obtenidos sobre desprofesionalización y (des)igualdades pueden ser de utilidad en análisis futuros que consideren la incidencia de las nuevas formas de acceso a la profesión y, de llevarse a la práctica, los cambios en la obligatoriedad de la colegiación para ejercer el derecho.

Palabras clave: análisis socioprofesional; abogacía; investigación psicosocial; investigación social; perfil sociodemográfico; formación profesional.

\section{Abstract. Changes, (in)equalities and daily life of law professionals}

The roots and the importance of the legal profession in Spanish society confer special interest to the knowledge about the current status of the profession and the changes observed among professionals in the field. The aim of this research note is to highlight indicators of deprofessionalization and gender (in)equalities in the professional practice of law. This objective was achieved by interviewing a sample of 562 law professionals (335 men and 227 women). Specifically, we describe and assess the following characteristics: demographic (gender, age), personal (the family unit and its relationship with the legal profession), and 
professional training (pre-and post-graduate education, entry to the profession and current practice). The analyses show that the youngest generation of lawyers is characterized by a majority of women and a smaller presence of family members in the legal profession. The deprofessionalization of the legal profession is evident in the differences between young and not-so-young attorneys as regards their entry into the profession and the positions they hold in law firms. (In)equalities are observed between male and female lawyers as regards training and the practice of different branches of law, as well as in the hours worked per week. The data obtained on deprofessionalization and (in)equalities can be useful in future analyses on the impact of new forms of entry to the profession and - if implemented - changes in compulsory licensing to practice law.

Keywords: socio-professional analysis; legal profession; psychosocial research; social research; socio-demographic characteristics; vocational training.

\section{Sumario}

1. Introducción 4. Conclusiones

2. Método Referencias bibliográficas

3. Resultados y discusión

\section{Introducción}

Abogadas y abogados forman parte de uno de los mayores grupos profesionales con estudios universitarios de la sociedad española. En el curso 2010-2011, los licenciados y las licenciadas en Derecho fueron el colectivo más numeroso de alumnos que culminaron sus estudios en las universidades españolas. Específicamente, se licenciaron 11.586 , lo que representa el 13,9\% del total de 82.864 nuevos profesionales universitarios (Instituto Nacional de Estadística, 2012).

El protagonismo y el peso de la abogacía en el conjunto del Estado han estimulado que, desde hace décadas, esta profesión haya sido objeto de estudio, lo cual ha coincidido con los primeros intentos de sistematización de los estudios socioprofesionales y la génesis de la sociología de las profesiones en España (Rodríguez Ávila, 2008; Sáez et al., 2009). Para Sáez et al. (2009), el interés por estos estudios puede explicarse por los paralelismos entre los cambios observados aquel entonces en las profesiones y los de la propia sociedad española de la época, lo que, a su vez, entendemos, condicionaría los tópicos que se han analizado.

Las temáticas de estudios sobre la abogacía (de cariz jurídico, sociológico o psicosocial) han sido muy variadas y de distinto calibre; esto es, hay desde contribuciones meramente descriptivas hasta elaboraciones sobre la situación del derecho, ya sea desde el punto de vista de la clientela real o potencial, letrados y letradas o analistas de la profesión. A título ilustrativo, respecto a cómo ve la ciudadanía al abogado, se valoran las imágenes globales que la sociedad tiene de dichos profesionales (Instituto de Investigaciones y Análisis Socio-jurídicos, 2003; Metroscopia, 2005, 2011; Ordre des Avocats à la Cour de Paris, 2002; Rojas, 2009), las percepciones puntuales de la confianza del público en los 
letrados (Ajuntament de Barcelona, 1998) o la responsabilidad que atribuye la ciudadanía a los abogados y a las abogadas cuando abordan temas de interés social (Centro de Investigaciones Sociológicas, 1998, 2001, 2002; Toharia, 1984). Por lo que se refiere a los estudios que analizan a la profesión a partir de sus protagonistas, están el estudio sobre la formación y/o los antecedentes familiares de los profesionales en el derecho (Balcells, 1974; Pestana, 2008; Soriano, Ruiz de la Cuesta y González-Tablas, 1987), las valoraciones de los abogados y las abogadas sobre aspectos puntuales de su práctica profesional (Instituto DYM, 2003) o los análisis del ejercicio de la abogacía en su conjunto (Balcells, 1975, 1978; Codina y Pestana, 2006; Metroscopia, 2008; Rodríguez Ávila, 2000, 2001). Por último, también destacan las reflexiones sobre el ejercicio profesional orientadas a describir fenómenos emergentes o escenarios de futuro de la abogacía (Aguilera, 1999; Angulo, 1994; Asociación Internacional de Jóvenes Abogados, 1983; Carnero et al., 1997; Círculo de Estudios Jurídicos, 1972; Cremades, 1995; Espinós, 1994; Ezama et al., 1997; Fédération des Barreaux d'Europe e Il-lustre Col-legi d'Advocats de Barcelona, 2004; Gutiérrez et al., 2002; Mullerat, 2001; Pérez Moreno, 2003; Stumpf et al., 1971) y los análisis comparativos sobre la percepción de la inserción laboral entre estudiantes universitarios de distintas carreras y zonas de Europa (Troiano, 2005).

Además de ser importantes, por aportar unos datos organizados y contrastados de la realidad de la profesión, los estudios anteriores, entre otras cosas, dan a conocer lo que Abel (1980) considera fundamental: saber qué hace, en el día a día, el abogado para el cliente. Es decir, estos estudios permiten estar al corriente de aspectos concretos del quehacer del profesional en un momento o en un periodo determinado y, dado que la realidad de la abogacía es tremendamente dinámica y compleja (Flood, 1991), es importante contar con estudios que informen y actualicen datos en torno a la profesión. En este sentido, además de analizar distintos aspectos del perfil humano del letrado o la letrada, en el presente trabajo se valorarán fenómenos tan debatidos en los últimos años como son la desprofesionalización de la abogacía y la incorporación de las mujeres en la misma.

La denominada «desprofesionalización de la abogacía» ha sido objeto de análisis dirigidos fundamentalmente a la pérdida de privilegios con respecto a épocas pasadas y a la proletarización de sus miembros, cambios que han afectado al conjunto de las profesiones en las últimas décadas (Guillén, 1990; Sánchez y Sáez, 2009). En el caso del derecho, en los alegatos sobre su desprofesionalización, conviven dos tendencias. La que aglutina posturas moderadas, asume la redefinición de la autonomía de la abogacía (su reprofesionalización: Powell, 1985) como parte de un proceso lógico de cambios que afectan al contexto en que se ejerce la profesión. Otra tendencia más radical es la que advierte lesiones en la autonomía de la profesión legal, como consecuencia de la reorganización de las funciones del letrado o la letrada y la segmentación de su trabajo (Abel, 1986; Brooks, 2011). Como un indicador de esta pérdida de autonomía, se ha destacado el aumento de los grandes despachos como fuente 
de empleo de abogados y abogadas, ya que el trabajo — asalariado- pasa a estar pautado por una corporación y no tanto por el propio criterio del profesional (Flood, 2011; Heinz et al., 2001; Zaragoza, 1975).

El incremento del número de abogadas en el contexto del ejercicio de la profesión se ha desarrollado en sincronía con la mayor presencia de mujeres en la vida pública (Fette, 2007), lo que ha traído consigo la introducción de nuevos valores en la concepción y el ejercicio del derecho (Anleu, 1992). No obstante, la mayor presencia de abogadas no parece que se haya acompañado de igualdad de condiciones de trabajo entre sexos. Así, por ejemplo, se han encontrado diferencias significativas entre letrados y letradas con respecto a la estructura y los usos de los tiempos profesional y personal (Codina y Pestana, 2006; Wallace, 1997, 1999). Estas diferencias parece que corroboran el aserto de Kay y Hagan (1998) sobre la adaptación de las abogadas a las expectativas de los despachos - y no al revés-, lo que implica que las mujeres deban anteponer el trabajo a otros ámbitos de la vida cotidiana. Entre nosotros, Rodríguez Ávila (2001: 303) ha dejado constancia de la abogacía como profesión «rejuvenecida y feminizada», es decir, integrada por profesionales predominantemente jóvenes y del sexo femenino. Así pues, el género —además de la edad - aparece como una variable a tener en cuenta al analizar el ejercicio profesional del derecho.

Con el objetivo de actualizar y complementar conocimientos sobre el ejercicio de la abogacía, en este trabajo nos proponemos mostrar las tendencias de cambio que se aprecian entre los profesionales ejercitantes en los perfiles demográfico, personal, de formación y ejercicio profesional. Los datos recogidos dibujan un mapa útil para identificar tendencias de cambio demográfico y personal, así como la intensidad con la que aparecen los fenómenos de desprofesionalización y de (des)igualdades de género en la abogacía.

\section{Método}

En el estudio, participaron 562 abogados y abogadas con más de un año de ejercicio profesional, seleccionados al azar a partir de la base de datos gestionada por el Departamento de Informática del Ilustre Colegio de Abogados de Barcelona (ICAB). Del total de 125.200 abogados y abogadas ejercitantes del Estado, el ICAB integra un 11,2\% del total. Es el segundo colegio más importante tras el de Madrid (cifras de diciembre de 2010: Consejo General de la Abogacía Española, 2011).

El grupo de participantes lo integraron 335 hombres (un 59,6\%) y 227 mujeres (un 40,4\%), de edades comprendidas entre los 23 y los 90 años $(M=39,76 ; D T=11,31)$. De acuerdo con los criterios de clasificación del propio ICAB, en el grupo estudiado, 343 (un 61,0\%) eran abogados y abogadas jóvenes (hasta los 39 años inclusive) y 219 (un 39,0\%) eran no jóvenes (de 40 y más años).

La información fue recogida a través de un protocolo de entrevista elaborado ad hoc por un grupo interdisciplinario de expertos. Dicho protocolo 
fue puesto a prueba y contrastado por una muestra de diez profesionales de la abogacía. La versión definitiva del instrumento estuvo compuesta mayoritariamente por preguntas cerradas y diseñado para ser usado de manera directiva.

Los aspectos contemplados en los ítems de la entrevista fueron: sexo; edad; estado civil; entorno familiar (hijos e hijas, antecedentes familiares vinculados al derecho y profesión de la pareja); experiencia profesional (inicio en la abogacía, años transcurridos como abogado o abogada ejercitante), y situación profesional actual (contexto en el que ejerce, horas trabajadas y dedicación a las distintas ramas del derecho - distinguiendo entre generalista, esto es, sin una especialización en particular-, dedicación a dos o tres ramas y especialista - con dedicación exclusiva a una rama de la profesión, a saber: civil, laboral, mercantil, penal, administrativa o tributaria).

Los profesionales y las profesionales seleccionados fueron contactados telefónicamente para concertar el día, la hora y el lugar de realización de la entrevista (con una duración aproximada de 25 a 30 minutos). Previo al inicio de la recolección de la información, concertadoras y entrevistadores recibieron dos sesiones de formación a cargo de los responsables del estudio - quienes, además, supervisaron todo el proceso de recogida de datos. En la información obtenida, no se observaron diferencias que pudieran vincularse a características individuales o estilos de trabajo de concertadoras y entrevistadores.

\section{Resultados y discusión}

\subsection{Perfil demográfico y personal de los profesionales de la abogacía}

El sexo, la edad, el estado civil o la estructura familiar son aspectos que nos acercan a la dimensión más personal del colectivo de abogados.

En relación con el sexo, globalmente considerado, el número de hombres es superior al de mujeres. No obstante, combinando las variables sexo y edad, se encuentran diferencias estadísticamente significativas $(p<0,000$ - ver figura 1$)$, según las cuales el número de abogadas es superior al de abogados entre los 25 y los 30 años; las proporciones se equilibran entre los 30 y los 35 años; a partir de los 35 años, el predominio de hombres aumenta, y con más de 60 años, en la muestra estudiada, no figura ninguna mujer. Estas diferencias también se observan al comparar profesionales jóvenes (menores de 40 años) y no jóvenes (con 40 y más años). Así, mientras que, entre los jóvenes, los porcentajes de mujeres y hombres son, respectivamente, el $50,4 \%$ y el $49,6 \%$, entre los no jóvenes sólo el $24,7 \%$ son mujeres $(p<0,000)$. 
Figura 1. Porcentaje de abogados según sexo y edad (en intervalos de cinco años) $(n=562)$

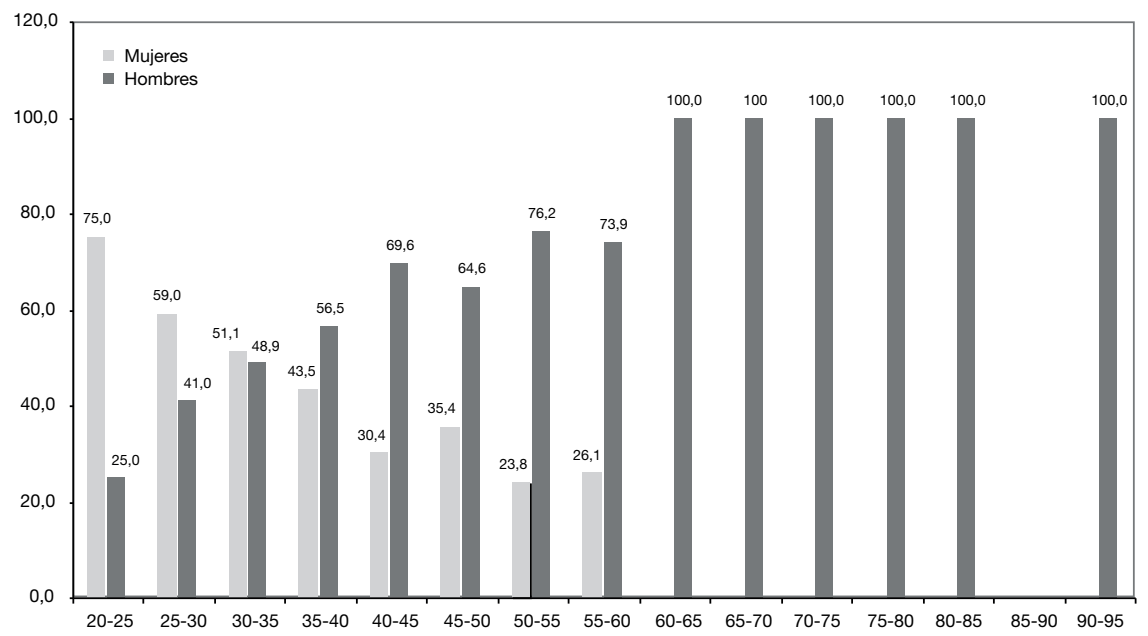

Fuente: elaboración propia.

Figura 2. Porcentaje de abogados jóvenes y no jóvenes según estado civil $(n=562)$

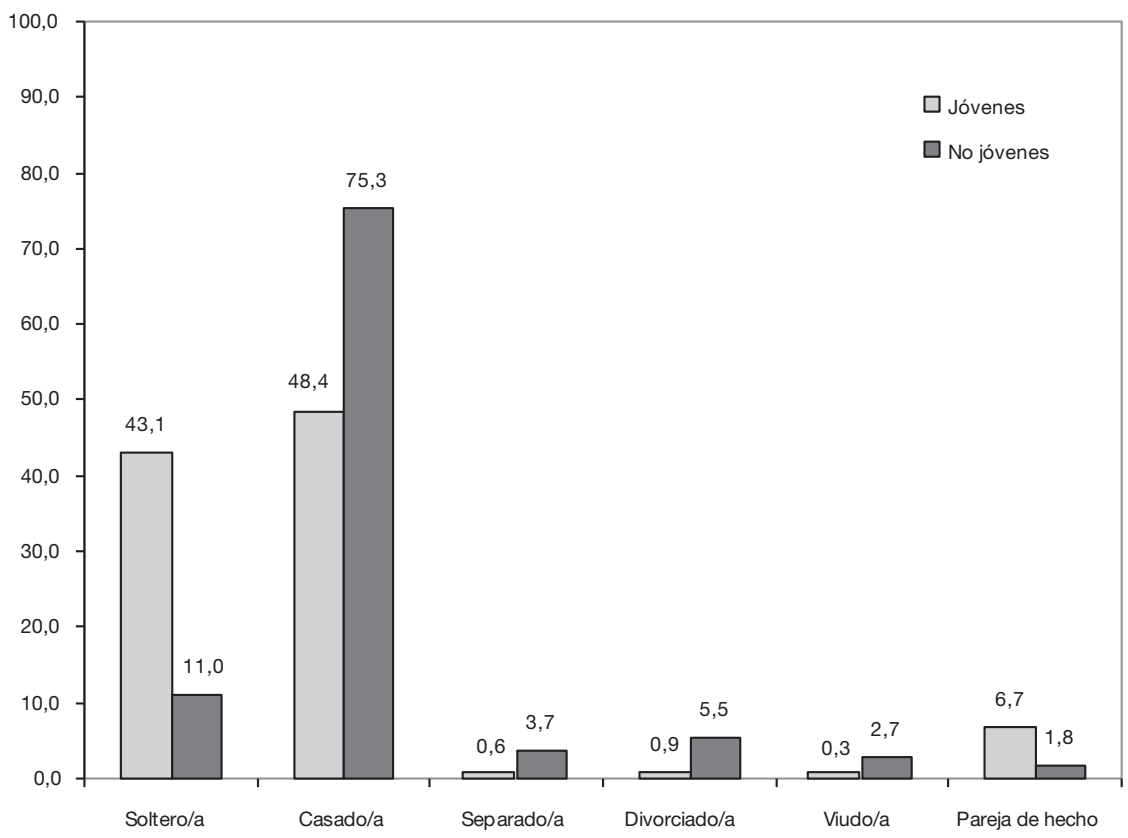

Fuente: elaboración propia. 
El estado civil predominante en el colectivo de abogados y abogadas es el de persona casada, con un porcentaje del 58,9\%. El siguiente estado civil más señalado es el de soltero o soltera, con una tercera parte de las personas entrevistadas (un 34,8\%). Con porcentajes inferiores al 5\%, están las siguientes situaciones: parejas de hecho (un 4,8\%), divorciados y divorciadas (un 2,7\%), separados y separadas (un 1,8\%) y viudos y viudas (un 1,2\%).

Entre el estado civil y la edad, se han encontrado diferencias significativas $(p<0,000)$. Como se puede ver en la figura 2 , en el conjunto de las personas mayores de 40 años, el hecho de estar casadas caracteriza a tres cuartas partes de este grupo (un $75,3 \%$ ) y el estar solteras, a un $11,0 \%$. En cambio, entre la juventud, el porcentaje de ambos estados civiles es bastante similar (esto es, un $48,4 \%$ de personas casadas y un $43,1 \%$ de solteras). Finalmente, aunque las proporciones son pequeñas, en la categoría "pareja de hecho" es interesante observar que hay cuatro veces más de jóvenes (un 6,7\%) que de no jóvenes (un $1,8 \%$ ).

En relación con la familia, los datos relativos a la descendencia muestran que prácticamente una mitad tiene hijos y la otra mitad no los tiene. En cifras, el 50,2\% tiene descendencia y el 49,8\%, no. Entre quienes tienen hijos, menos de la mitad tiene dos (un 44,3\%) y poco más de una cuarta parte tiene uno (un 28,7\%). Situaciones menos frecuentes son profesionales con tres hijos (un $18,4 \%$ ), cuatro (un $6,4 \%$ ), cinco (un $1,1 \%$ ), seis (un $0,7 \%$ ) o siete (un $0,4 \%)$. Según la edad del profesional, hay diferencias significativas con respecto al promedio de hijos $(p<0,000)$. Así, los jóvenes tienen una $M=0,5(D T=$ $0,9)$ y los no jóvenes, una $M=1,8(D T=1,3)$. Por lo que se refiere al sexo, también se observan diferencias significativas. Entre los jóvenes, ellos tienen de descendencia una $M=0,6(D T=0,9)$ y ellas una $M=0,4(D T=0,9)(p<$ $0,086)$. Entre los no jóvenes, con respecto al número de hijos, los datos señalan una $M=2,0(D T=1,3)$ para los abogados y una $M=1,3(D T=1,0)$ para las abogadas $(p<0,000)$.

\subsection{Familiares en el ámbito de la abogacía y el tópico de la "saga»}

Al considerar el entorno familiar de los profesionales de la abogacía por la naturaleza del parentesco, diferenciamos dos esferas en las que se pueden encontrar profesionales del ámbito jurídico: una es la de la familia de procedencia, y otra, la de la pareja del profesional.

Por lo que se refiere a la primera esfera, el 31,0\% tiene familiares vinculados al mundo del derecho. $\mathrm{Al}$ analizar esta variable en relación con la edad de las personas entrevistadas, se observa que entre los abogados y las abogadas jóvenes, el 28,3\% tiene familiares en la profesión, porcentaje que sube al 35,2\% en el grupo de no jóvenes $(p<0,085)$. Para más detalle, entre los profesionales con familiares en el campo del derecho, un 59,2\% tiene un familiar; un 27,0\%, dos; un $9,8 \%$, tres, y excepcionales son los casos de cuatro y cinco familiares (respectivamente, el 1,7\% y el 1,1\% —el 1,1\% restante no especificó cuántos familiares tenía en la profesión). 
En la otra esfera, la relativa a la pareja de los entrevistados, casi un $25 \%$ tiene alguna vinculación con el mundo del derecho. Entre los profesionales con pareja (409, que equivalen a un $71,5 \%$ del total de 562), la profesión de ésta fue descrita en los términos y con los porcentajes que se indican a continuación: abogado o abogada, el 18,8\%; abogado o abogada y otra profesión no jurídica, el 1,2\%; otras profesiones jurídicas, el 3,7\% —las proporciones restantes correspondieron a aquellas personas cuya pareja tiene una o más profesiones no vinculadas al derecho (un $74,6 \%$ ) y a quienes no especificaron la profesión de su pareja (un 1,7\%).

\subsection{Formación profesional: básica y especializada}

Tanto en la abogacía como en otros ámbitos, la formación de los profesionales ofrece datos tan interesantes como escasos - de ahí su interés particular. En concreto, en este trabajo, se han diferenciado dos grandes etapas: la básica, que lleva a obtener la licenciatura en Derecho, y la posterior a la licenciatura, que incluye el doctorado y los cursos de especialización o formación en distintas ramas del derecho, de las ciencias sociales u otros ámbitos disciplinarios.

Por lo que se refiere a la formación básica y a los centros universitarios donde se han realizado los estudios, se observa homogeneidad. Casi la totalidad de las personas entrevistadas del ICAB ha obtenido el título de licenciado en Derecho en una universidad española (un 99,2\%). En términos geográficos, un $91,5 \%$ se ha licenciado en Cataluña; un 1,1\%, en Madrid, y un 3,4\%, en la Universidad Nacional de Educación a Distancia (UNED). Por lo que se refiere específicamente a los centros universitarios catalanes, por la Universidad de Barcelona, se ha licenciado un 69,6\%; por la Autónoma de Barcelona, un 10,7\%; por la Pompeu Fabra, un 4,8\%; por la Abat Oliba CEU, un 3,9\%, y por la Ramon Llull, un 1,2\%.

En la formación posterior a la licenciatura, se observa una diversidad considerable. A pesar de que, de entrada, las posibilidades de formación se limitan a dos orientaciones no excluyentes entre sí —estudios de doctorado y estudios de postgrado o máster-, dentro de cada una de ellas las alternativas se amplían.

Los estudios de doctorado han sido seguidos por un 15,4\% de los entrevistados. Este porcentaje lo integra un 3,7\% que ha hecho los cursos de doctorado, un $9,0 \%$ que ha obtenido el certificado de suficiencia investigadora o el diploma de estudios avanzados y un $2,7 \%$ que ha defendido su tesis doctoral $y$, por lo tanto, es doctor o doctora.

Por lo que respecta a los cursos de especialización, un $67,9 \%$ de la muestra afirma que había seguido este tipo de formación. Para más precisión, un $33,5 \%$ dijo que había realizado un curso; un $19,2 \%$, dos; un $8,7 \%$, tres; un $3,4 \%$, cuatro, y un $1,3 \%$, entre cinco y siete (el 1,8\% restante no especificó los estudios realizados).

El contenido general de los cursos mencionados se analizó a partir de una clasificación preestablecida por el sistema de expertos, la cual consideraba las siguientes ramas del derecho: administrativo, civil, internacional, laboral, mer- 
Tabla 1. Cursos de especialización según la edad y el sexo de los profesionales y las profesionales

\begin{tabular}{|c|c|c|c|c|c|c|c|c|c|c|c|}
\hline \multirow{3}{*}{$\begin{array}{l}\text { Rama de los cursos } \\
\text { de especialización }\end{array}$} & \multirow{3}{*}{$\begin{array}{c}\% \\
(n=562)\end{array}$} & \multicolumn{5}{|c|}{$\begin{array}{l}\text { Porcentaje de abogados } \\
\text { y abogadas por edades }\end{array}$} & \multicolumn{5}{|c|}{$\begin{array}{l}\text { Porcentaje de abogados } \\
\text { y abogadas por sexos }\end{array}$} \\
\hline & & \multicolumn{2}{|c|}{$\begin{array}{c}\text { Jóvenes } \\
\text { (hasta } 39 \text { años) }\end{array}$} & \multicolumn{2}{|c|}{$\begin{array}{c}\text { No jóvenes } \\
\text { (40 y más años) }\end{array}$} & \multirow[b]{2}{*}{$\chi^{2}$} & \multicolumn{2}{|c|}{ Hombres } & \multicolumn{2}{|c|}{ Mujeres } & \multirow[b]{2}{*}{$\chi^{2}$} \\
\hline & & $n$ & $\%$ & $n$ & $\%$ & & $\mathrm{n}$ & $\%$ & $\mathrm{~N}$ & $\%$ & \\
\hline Derecho administrativo & 3,9 & 15 & 68,2 & 7 & 31,8 & $15,81^{\star \star \star}$ & 17 & 77,3 & 5 & 22,7 & $6,70^{\star \star}$ \\
\hline Derecho civil & 12,3 & 36 & 52,2 & 33 & 47,8 & $23,23^{\star \star \star}$ & 34 & 49,3 & 35 & 50,7 & $5,01^{*}$ \\
\hline Derecho internacional & 5,5 & 19 & 61,3 & 12 & 38,7 & $16,20^{\star \star \star}$ & 15 & 48,4 & 16 & 51,6 & 3,90 \\
\hline Derecho laboral & 6,4 & 28 & 77,8 & 8 & 22,2 & $17,85^{\star \star \star}$ & 17 & 47,2 & 19 & 52,8 & 4,45 \\
\hline Derecho mercantil & 9,6 & 27 & 50,0 & 27 & 50,0 & $23,14^{\star \star \star}$ & 30 & 63,0 & 20 & 37,0 & 3,67 \\
\hline Derecho penal & 3,4 & 14 & 73,7 & 5 & 26,3 & $16,20^{\star \star \star}$ & 13 & 68,4 & 6 & 31,6 & 3,85 \\
\hline Derecho tributario & 10,5 & 34 & 57,6 & 25 & 42,4 & $18,19^{\star \star \star}$ & 41 & 69,5 & 18 & 30,5 & $7,17^{\star \star}$ \\
\hline Transversales & 28,3 & 116 & 73,0 & 43 & 27,0 & $20,33^{\text {***}}$ & 93 & 58,5 & 66 & 41,5 & 2,99 \\
\hline Àreas distintas al derecho & 12,6 & 37 & 52,1 & 34 & 47,9 & $23,56^{\star \star \star}$ & 41 & 57,7 & 30 & 42,3 & 2,81 \\
\hline No precisa & 10,1 & 39 & 68,4 & 18 & 31,6 & $15,87^{\star \star \star}$ & 33 & 57,9 & 24 & 42,1 & 2,82 \\
\hline No ha realizado cursos & 32,2 & - & - & - & - & - & - & - & - & - & - \\
\hline
\end{tabular}

Nota. Las personas entrevistadas podían señalar cursos en diversas especialidades. ${ }^{\star} p<0,010$; ${ }^{\star \star} p<0,050 ;{ }^{\star \star \star} p<0,000$.

Fuente: elaboración propia.

cantil, penal y tributario. No obstante, ciertas particularidades de la información facilitada aconsejaron añadir tres categorías: estudios transversales (cursos que comprenden diversas ramas del derecho, p. e.: combinaciones entre las ramas administrativa, mercantil y tributaria, o bien estudios como los de las escuelas de práctica jurídica); estudios en áreas distintas al derecho, y, finalmente, una categoría de estudios no especificados.

Tal como se puede ver en la tabla 1 , en general, sobresalen las especializaciones transversales (que contemplan diversas ramas del derecho: 28,3\%). A continuación, dentro de las ramas del derecho sustantivo, destacan los cursos sobre derecho civil (un 12,3\%), tributario (un 10,5\%) y mercantil (un 9,6\%). Junto a estos porcentajes, sin embargo, es de destacar que un $12,6 \%$ se ha interesado por formarse en materias distintas al derecho (formación que suele referirse a ciencias sociales y humanas).

En relación con la edad y el sexo, se encuentran diferencias significativas en los estudios de especialización (tabla 1). En líneas generales, los profesionales y las profesionales jóvenes se han decantado por una formación en derecho laboral (un 77,8\%), penal (un 73,7\%) y administrativo (un 68,2\%); y comparten sensibilidades con los abogados y las abogadas no jóvenes en el campo del derecho mercantil (un 50,0\%) y la formación en ámbitos de conocimiento distintos al derecho (un 52,1\%). Entre los profesionales y las profesionales no jóvenes, de manera específica y particular, despunta un interés especial por el derecho tributario (un 42,4\%). Por lo que se refiere a la relación entre el sexo y el contenido de los cursos posteriores a la licenciatura, se observan tanto similitudes como diferencias. Así, los cursos de derecho civil son seguidos equitativamente por abogadas (un 50,7\%) y abogados (un 49,3\%), y lo mismo 
ocurre con los de derecho internacional (un 51,6\% de mujeres y un 48,4\% de hombres). En cambio, los abogados sobresalen significativamente en los cursos de derecho administrativo (un $77,3 \%$ ) y tributario (un $69,5 \%$ ).

\subsection{Ejercicio de la abogacía: inicios y situación actual}

Con respecto al ejercicio del derecho, atendemos al inicio en la abogacía (en relación con la finalización de la licenciatura, la situación laboral inicial y la retribución percibida), el contexto en que se ejerce la profesión en la actualidad (en particular, las características del despacho y su situación en el mismo), las horas trabajadas a la semana y la dedicación a las distintas áreas del derecho.

Tal como puede verse en la tabla 2, el inicio en el ejercicio de la profesión, para el 58,0\% de la muestra, fue al año siguiente de haber obtenido la licenciatura, y para el 36,3\%, el mismo año en que culminó los estudios. Sólo un $5,7 \%$ había comenzado a involucrarse en el trabajo de letrado o letrada con anterioridad a la licenciatura. Al comparar estos datos según la edad de las personas entrevistadas, se observan diferencias significativas $(p<0,047)$. Específicamente, en el grupo de jóvenes, el 61,2\% empezó a ejercer a partir del año siguiente de licenciarse y el 32,4\% lo hizo el mismo año en que culminó sus estudios. En cambio, entre los abogados y las abogadas no jóvenes, el 53,0\% comenzó su ejercicio profesional a partir del año siguiente de haber terminado sus estudios y el $42,5 \%$, en el mismo año en que obtuvo la licenciatura.

$\mathrm{Al}$ comenzar a ejercer la abogacía, la situación laboral más frecuente es por cuenta ajena, específicamente éste es el caso del $59,4 \%$ de las personas entrevistadas, seguidas por el $30,2 \%$ que lo ha hecho por cuenta propia. Estos valores cambian de manera significativa según la edad de los abogados y las abogadas $(p<0,000)$. Así, el 66,5\% del grupo de jóvenes comenzó en la abogacía por cuenta ajena, y menos de una cuarta parte (un 24,2\%), por cuenta propia. En el caso de los no jóvenes, la proporción de letrados y letradas que se iniciaron en la profesión por cuenta ajena y por cuenta propia fueron, respectivamente, un $48,4 \%$ y un $39,7 \%$.

En los inicios como abogado o abogada, el tipo de retribución percibida fue constante en el $40,8 \%$ de los casos y aleatoria en el $28,8 \%$. Destaca, sin embargo, que el $22,4 \%$ no obtuvo retribución alguna cuando comenzó a ejercer. Al comparar jóvenes y no jóvenes, se observan diferencias significativas ( $p$ $<0,000$ ), según las cuales el $52,2 \%$ de los abogados y las abogadas menores de 40 años tuvo una retribución constante y el 25,9\%, nula. En los letrados y letradas de 40 y más años, los porcentajes de ambas modalidades de retribución fueron, respectivamente, un $41,1 \%$ y un $16,9 \%$, entre ellos destaca un $40,6 \%$ que percibió retribuciones de manera aleatoria por su trabajo.

Por lo que se refiere a la situación laboral actual de las personas entrevistadas (tabla 3), ésta se desarrolla mayoritariamente en el seno de un despacho profesional (un 74,4\%), y tiene un peso menor el trabajo en administraciones, empresas u otros contextos no especificados (un 25,6\%). Por lo que respecta a los despachos profesionales, es interesante valorar la posición de las abogadas 
Tabla 2. Inicios en la abogacía según la edad de los profesionales

\begin{tabular}{|c|c|c|c|c|c|c|c|}
\hline & \multirow{3}{*}{$\begin{array}{c}2 \\
\% \\
(n=562)\end{array}$} & \multicolumn{6}{|c|}{ Porcentaje de abogados y abogadas por edades } \\
\hline & & \multicolumn{2}{|c|}{$\begin{array}{c}\text { Jóvenes } \\
\text { (hasta } 39 \text { años) }\end{array}$} & \multicolumn{2}{|c|}{$\begin{array}{c}\text { No jóvenes } \\
\text { (40 y más años) }\end{array}$} & \multirow[b]{2}{*}{$\chi^{2}$} & \multirow[b]{2}{*}{$p$} \\
\hline & & $n$ & $\%$ & $n$ & $\%$ & & \\
\hline \multicolumn{8}{|l|}{ Momento de inicio } \\
\hline El mismo año de la licenciatura & 36,3 & 111 & 32,4 & 93 & 42,5 & & \\
\hline Antes de la licenciatura & 5,7 & 22 & 6,4 & 10 & 4,6 & & \\
\hline Después de la licenciatura & 58,0 & 210 & 61,2 & 116 & 53,0 & 6,13 & 0,047 \\
\hline \multicolumn{8}{|l|}{ Situación laboral inicial } \\
\hline Cuenta propia & 30,2 & 83 & 24,2 & 87 & 39,7 & & \\
\hline Cuenta ajena & 59,4 & 228 & 66,5 & 106 & 48,4 & & \\
\hline Cuenta propia y cuenta ajena & 7,8 & 24 & 7,0 & 20 & 9,1 & & \\
\hline Otras NE & 2,5 & 8 & 2,3 & 6 & 1,1 & 18,86 & 0,000 \\
\hline \multicolumn{8}{|l|}{ Retribución al comenzar } \\
\hline Ninguna & 22,4 & 89 & 25,9 & 37 & 16,9 & & \\
\hline Aleatoria & 28,8 & 73 & 21,3 & 89 & 40,6 & & \\
\hline Constante & 40,8 & 179 & 52,2 & 91 & 41,1 & & \\
\hline Aleatoria y constante & 0,7 & 2 & 0,6 & 2 & 0,4 & 25,60 & 0,000 \\
\hline
\end{tabular}

Fuente: elaboración propia.

y los abogados dentro del mismo en función del tamaño del despacho y la compartición o no del despacho.

En función del número de abogados y de abogadas (entre paréntesis), clasificamos los despachos como: pequeño (1-4), mediano (5-9) o grande (10 y más). Del total de entrevistados, cuatro de cada diez trabaja en un despacho pequeño (un 42,7\%), y son similares las proporciones de letrados y letradas que lo hacen en despachos medianos (un 15,7\%) y grandes (un 14,8\%). Al comparar esta variable según la edad de las personas participantes, se observan diferencias significativas $(p<0,000)$ : mientras las proporciones de abogados y abogadas jóvenes es de un 37,0\% en despachos pequeños, un 17,5\% en medianos y un 19,2\% en grandes, entre los no jóvenes, poco más de la mitad (un $51,6 \%)$ trabaja en despachos pequeños; aproximadamente uno de cada diez (un 12,8\%), en despachos medianos, y excepcionalmente ejercen su profesión en despachos grandes (un 3,0\%).

El tipo de despacho en que se desarrolla la labor profesional de las personas entrevistadas es el compartido (un 61,0\%). Si bien esta modalidad es la más habitual en los dos grupos de edad considerados, existen diferencias significativas $(p<0,025)$ entre jóvenes y no jóvenes. En concreto, comparte despacho el $64,7 \%$ de menores de 40 años y el $55,7 \%$ de 40 y más años. Respecto al ejercicio profesional en despacho no compartido, en esta modalidad encontramos un $16,9 \%$ de no jóvenes y un $9,0 \%$ de jóvenes.

La posición que ocupan los letrados y las letradas en el despacho, en el $43,6 \%$ del total de personas entrevistadas, es la de titular, socio o asociado. En relación con esta condición, se observan diferencias significativas según la 
Tabla 3. Situación laboral actual en la abogacía según la edad de los profesionales y las profesionales

\begin{tabular}{|c|c|c|c|c|c|c|c|}
\hline & \multirow{3}{*}{$\begin{array}{c}\% \\
(n=562)\end{array}$} & \multicolumn{6}{|c|}{ Porcentaje de abogados y abogadas por edades } \\
\hline & & \multicolumn{2}{|c|}{$\begin{array}{c}\text { Jóvenes } \\
\text { (hasta } 39 \text { años) } \\
\end{array}$} & \multicolumn{2}{|c|}{$\begin{array}{c}\text { No jóvenes } \\
\text { (40 y más años) }\end{array}$} & \multirow[b]{2}{*}{$\chi^{2}$} & \multirow[b]{2}{*}{$p$} \\
\hline & & $n$ & $\%$ & $n$ & $\%$ & & \\
\hline \multicolumn{8}{|l|}{$\begin{array}{l}\text { Contexto actual de la actividad } \\
\text { como abogado o abogada }\end{array}$} \\
\hline Despacho de abogados & 74,4 & 258 & 75,2 & 160 & 73,1 & & \\
\hline Administraciones y empresas & 17,3 & 62 & 18,1 & 35 & 16,0 & & \\
\hline Otros NE & 8,4 & 23 & 6,7 & 24 & 11,0 & 3,31 & 0,191 \\
\hline \multicolumn{8}{|l|}{$\begin{array}{l}\text { Número de abogados y abogadas } \\
\text { en el despacho }\end{array}$} \\
\hline $1-4$ & 42,7 & 127 & 37,0 & 113 & 51,6 & & \\
\hline $5-9$ & 15,7 & 60 & 17,5 & 28 & 12,8 & & \\
\hline 10 y más & 14,8 & 66 & 19,2 & 17 & 3,0 & & \\
\hline NE & 1,2 & 5 & 1,5 & 2 & 0,9 & & \\
\hline No trabaja en despacho & 25,6 & 85 & 24,8 & 59 & 26,9 & 21,02 & 0,000 \\
\hline \multicolumn{8}{|l|}{ Tipo de despacho } \\
\hline No compartido & 12,1 & 31 & 9,0 & 37 & 16,9 & & \\
\hline Compartido & 61,0 & 222 & 64,7 & 121 & 55,3 & & \\
\hline NE & 1,2 & 5 & 1,5 & 2 & 0,9 & & \\
\hline No trabaja en despacho & 25,6 & 85 & 24,8 & 59 & 26,9 & 9,34 & 0,025 \\
\hline \multicolumn{8}{|l|}{ Posición en el despacho } \\
\hline Titular, socio o asociado & 43,6 & 112 & 32,7 & 133 & 60,7 & & \\
\hline No titular, no socio o no asociado & 29,4 & 140 & 40,8 & 25 & 11,4 & & \\
\hline $\mathrm{NE}$ & 1,4 & 6 & 1,7 & 2 & 0,4 & & \\
\hline No trabaja en despacho & 25,6 & 85 & 24,8 & 59 & 26,9 & 64,42 & 0,000 \\
\hline
\end{tabular}

Fuente: elaboración propia.

edad de las letradas y los letrados $(p<0,000)$ : mientras que el $60,7 \%$ de los no jóvenes es titular, socio o asociado, sólo el 32,7\% de los jóvenes y las jóvenes goza de esta condición.

Las horas trabajadas a la semana por las personas entrevistadas oscilan entre 7 y $90(M=45,49$ y $D T=45,49)$-información no tabulada. En esta variable no se observaron diferencias significativas por edad, pero sí por sexo $(t=3,70$; $p<0,000$ ), puesto que daba como resultado más horas trabajadas en el caso de los hombres $(M=46,7$ y $D T=10,2)$ con respecto a las mujeres $(M=43,5$ y $D T=9,4)$.

Finalmente, con respecto a la dedicación actual a las ramas del derecho (tabla 4), la mayoría de las diferencias significativas se observaron entre abogadas y abogados. Ellos - más que ellas - suelen ejercer como abogados generalistas $(p<0,050)$ o dedicarse a las ramas administrativa $(p<0,000)$, mercantil $(p<0,050)$ y tributaria $(p<0,050)$. Entre generaciones, sólo se observó que los jóvenes llevan asuntos sobre derecho tributario con mayor asiduidad $(p<$ $0,010)$ que los no jóvenes. 
Tabla 4. Dedicación actual a ramas del derecho según la edad y el sexo de las profesionales y los profesionales

\begin{tabular}{|c|c|c|c|c|c|c|c|c|c|c|c|}
\hline \multirow[b]{3}{*}{ Ramas del derecho } & \multirow{3}{*}{$\begin{array}{c}\% \\
(n=562)\end{array}$} & \multicolumn{5}{|c|}{ Abogados y abogadas por edad } & \multicolumn{5}{|c|}{ Abogados y abogadas por sexo } \\
\hline & & \multicolumn{2}{|c|}{$\begin{array}{c}\text { Jóvenes } \\
\text { (hasta } 39 \text { años) }\end{array}$} & \multicolumn{2}{|c|}{$\begin{array}{c}\text { No jóvenes } \\
\text { (40 y más años) }\end{array}$} & \multirow[b]{2}{*}{$T$} & \multicolumn{2}{|c|}{ Hombres } & \multicolumn{2}{|c|}{ Mujeres } & \multirow[b]{2}{*}{$t$} \\
\hline & & $M$ & $D T$ & $M$ & $D T$ & & $M$ & DT & $M$ & $D T$ & \\
\hline Derecho generalista & 85,7 & 2,6 & 0,9 & 2,6 & 1,0 & $-0,39$ & 2,7 & 1,0 & 2,5 & 0,9 & $2,46^{\star *}$ \\
\hline Derecho administrativo & 82,7 & 2,0 & 0,9 & 2,1 & 1,0 & $-1,00$ & 2,2 & 0,9 & 1,9 & 0,9 & $3,70^{\star \star \star}$ \\
\hline Derecho civil & 93,4 & 3,1 & 0,9 & 3,2 & 0,9 & $-0,58$ & 3,1 & 0,9 & 3,2 & 0,9 & $-1,47$ \\
\hline Derecho internacional & 100,0 & 1,4 & 0,8 & 1,4 & 0,8 & 0,29 & 1,4 & 0,8 & 1,4 & 0,8 & $-0,14$ \\
\hline Derecho laboral & 100,0 & 1,7 & 1,0 & 1,6 & 1,0 & 1,10 & 1,6 & 0,9 & 1,7 & 1,1 & $-1,37$ \\
\hline Derecho mercantil & 100,0 & 2,2 & 1,1 & 2,2 & 1,1 & 0,81 & 2,3 & 1,1 & 2,0 & 1,1 & $2,63^{\star \star}$ \\
\hline Derecho penal & 100,0 & 1,9 & 1,1 & 1,8 & 1,0 & 0,49 & 1,9 & 1,1 & 1,8 & 1,0 & 0,80 \\
\hline Derecho tributario & 100,0 & 1,7 & 1,0 & 1,6 & 0,9 & $1,78^{\star}$ & 1,8 & 1,0 & 1,6 & 0,9 & $2,36^{\star \star}$ \\
\hline Otras ramas del derecho & 80,7 & 1,8 & 1,2 & 1,7 & 1,2 & 0,71 & 1,7 & 1,2 & 1,8 & 1,3 & $-1,43$ \\
\hline
\end{tabular}

Nota. Las personas entrevistadas podían señalar diversas especialidades. Las opciones de respuesta de estas preguntas fueron: 1, nada; 2, poco; 3 , bastante; 4 , mucho. ${ }^{*} p<0,010 ;{ }^{* \star} p<0,050 ;{ }^{* \star \star} p<0,000$.

Fuente: elaboración propia.

\section{Conclusiones}

El análisis de los perfiles demográfico, personal, de formación y ejercicio profesional de las abogadas y los abogados ejercitantes del ICAB muestran que la abogacía es una profesión que ha seguido un proceso de transformación en el que se advierten indicadores de desprofesionalización y de diferencias entre sexos.

Como indicadores de este proceso de transformación, se constata la mayor presencia de mujeres entre las generaciones más jóvenes de profesionales, hecho importante al haber predominado históricamente los hombres en la abogacía. Sobre el entorno familiar de los letrados y las letradas, es importante subrayar, por una parte, las afinidades de los familiares en el mundo del derecho (con un $31,0 \%$ con antecedentes en la abogacía y un $23,7 \%$ cuya pareja está vinculada a la profesión legal). En relación con la formación conducente a la licenciatura, se observan signos de escasa movilidad; con respecto a la formación de postgrado, los datos obtenidos sugieren la necesidad de atender, en estudios futuros, al ajuste entre la oferta de estudios de tercer ciclo y las demandas - necesidades, problemas - del día a día del profesional del derecho.

La desprofesionalización de la abogacía se pone de manifiesto en las diferencias entre jóvenes y no jóvenes con respecto a los inicios en el ejercicio de la profesión y su posición actual en el contexto del despacho profesional. Esta desprofesionalización se advierte entre las abogadas y los abogados jóvenes (con escasa proporción de familiares en el derecho) que se inician más tarde en el ejercicio profesional, mayoritariamente por cuenta ajena y percibiendo retribuciones que, en la mitad de los casos, son constantes y, en uno de cada cuatro casos, nulas. Asimismo, pueden considerarse indicadores de la desprofesionalización: la posición que ocupan los jóvenes abogados y abogadas en el 
despacho, que, predominantemente, es la de no titular, no socio o no asociado, y el tipo de despacho que contrata a uno de cada cinco letrados y letradas jóvenes, que es un despacho grande (de diez o más abogados y abogadas), en el que no parece predominar el trabajo intergeneracional con las profesionales y los profesionales de 40 años y más (de los que sólo un 3,0\% ejerce en despachos grandes).

Por lo que se refiere a las (des)igualdades entre abogadas y abogados, los datos de nuestro estudio no muestran diferencias significativas en perjuicio de las mujeres, pero sí apuntan dos indicadores que deberían ser analizados con más profundidad en estudios sucesivos. Por una parte, las diferencias en la formación y el ejercicio actual de las ramas del derecho muestran la necesidad de valorar si la oferta formativa favorece la conciliación igualitaria de los tiempos personales y laborales en mujeres y hombres. Por otra parte, las diferencias en el número de horas trabajadas entre abogados y abogadas sugieren que es preciso seguir analizando esta realidad y su posible incidencia en las oportunidades futuras de desarrollo profesional de las abogadas.

En definitiva, los datos sobre el profesional o la profesional de la abogacía nos muestran unos perfiles cuyos trazos sucesivos irán dibujando la compleja realidad del ejercicio del derecho. En investigaciones futuras, será necesario considerar, por ejemplo, abogados o abogadas adscritos a otros colegios (y, por extensión, vinculados a otros entornos personales y profesionales). Asimismo, sería interesante comparar el perfil de los abogados y las abogadas con otros colectivos profesionales e incluso con los de la población en general. Esto permitiría contrastar características de la muestra aquí analizada, como el 43,1\% de solteros y solteras entre los menores de 40 años o el $49,8 \%$ de profesionales sin descendencia. Finalmente, mencionar que, en la agenda futura de estudios de la abogacía, será necesario considerar la incidencia de las nuevas formas de acceso a la profesión y, de llevarse a la práctica, los cambios en la obligatoriedad de la colegiación para ejercer el derecho. En uno y otro caso, abogadas y abogados se encontrarán en una posición intersticial, por ser socialmente inducidos a unos modos de ejercer la profesión y por ser inductores de cambios que repercuten sobre sus prestaciones a la sociedad.

\section{Nota}

Los autores agradecen los comentarios realizados por dos revisores anónimos a la primera versión de esta nota de investigación. La realización de esta contribución ha contado con la colaboración del Comisionado para Universidades e Investigación del DIUE de la Generalidad de Cataluña, a través de PsicoSAO (2009 SRG 210; grupo de investigación consolidado en psicología social, ambiental y de las organizaciones). 


\section{Referencias bibliográficas}

ABEL, Richard. L. (1980). «Redirecting social studies of law». Law and Society Review, 14 (3), 805-829.

- (1986). «The transformation of the American Legal Profession». Law and Society Review, 20 (1), 7-17.

Aguilera Luna, Fernando (1999). Reflexiones sobre la abogacía para jóvenes abogados. Sevilla: Tecnographic.

Ajuntament De Barcelona (1998). Enquesta de qualitat de la ciutat. Barcelona: Institut Municipal d'Informàtica, Estudis i Avaluació.

ANgulo RodrígueZ, Luis de (1994). «El ejercicio de la abogacía en los albores del año 2000». En: GUTIÉRREZ-Alviz CONRADI, Faustino (ed.). El abogado: formación, deontología y formación del despacho profesional. Pamplona: Aranzadi.

ANLEU, Sharyn L. Roach (1992). "Women in Law: Theory, Research and Practice». Australian and New Zealand Journal of Sociology, 28 (3), 391-410.

ASOCIACIÓN INTERNACIONAL DE JÓVENES AbOGADOS (1983). "Cambiar la estructura de la profesión para responder a las necesidades de la clientela del mañana». Anuario de Sociología y Psicología Jurídicas, 10, 71-114.

BALCELlS, José (1974). «Encuesta sobre la situación social de la abogacía en Barcelona». Anuario de Sociología y Psicología Jurídicas, 1, 65-123.

- (1975). «Encuesta sobre las opiniones de la abogacía barcelonesa en materia profesional familiar y política». Anuario de Sociología y Psicología Jurídicas, 2, 105-150.

- (1978). «Satisfacciones e insatisfacciones de la abogacía barcelonesa respecto del ejercicio profesional». Anuario de Sociología y Psicología Jurídicas, 5, 69-103.

BROOKS, Robert A. (2011). Cheaper by the hour: Temporary Lawyers and the Deprofessionalization of the Law. Filadelfia, PA: Temple University Press.

Carnero, Ana Isabel; Montalvo, Eduardo y Velilla, Lourdes (1997). «El abogado/a en los servicios sociales generales». Intervención Psicosocial, VI (17), 217-226.

Centro de Investigaciones Sociológicas (1998). Estudio no 2.278. Barómetro de febrero. Madrid: CIS.

- (2001). Estudio no 2.411. Barómetro de marzo. Madrid: CIS.

- (2002). Estudio no 2.448. Barómetro de febrero. Madrid: CIS.

CírCulo de Estudios Jurídicos (1972). La abogacía en el año 2000: Coloquios organizados por el Círculo de Estudios Jurídicos de Madrid, los dias 25 y 26 de febrero, y 22 y 23 de marzo de 1971. Madrid: Círculo de Estudios Jurídicos.

CODina, Nuria y PestanA, José Vicente (2006). «Un análisis socioprofesional de la abogacía: ¿ruptura de estereotipos? ¿perfiles borrosos?». Revista de Psicología Social Aplicada, 16 (2), 303-321.

Consejo General de la Abogacía Española (2011). Censo de abogados por colegios (actualizado diciembre de 2010) [en línea]. Madrid: CGAE. <http://www.cgae.es/ portalCGAE/printPortal.do?urlPagina=S001011003/es_ES.html> [Consulta: 25 agosto 2011].

CREMADES, Bernardo M. (1995). «La abogacía, una profesión en cambio». Actualidad Jurídica Aranzadi, V (183), 1-4.

EsPINÓS, Álvaro (1994). «Evolució de la professió jurídica per tal d'adaptar-la a la prestació dels serveis que demana la societat d'avui». IURIS, 3, 211-222.

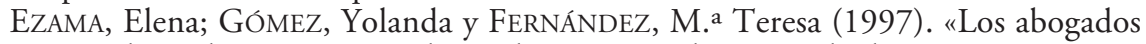
ante la mediación: Un estudio exploratorio en el Principado de Asturias». Anuario de Psicología Jurídica, 7, 121-147. 


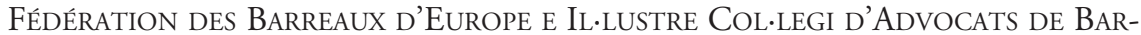
CELONA (2004). Encuesta a colegios de abogados pertenecientes a la Fédération des Barreaux d'Europe. Una primera aproximación (informe técnico no publicado). Barcelona: ICAB.

Fette, Julie (2007). «Pride and Prejudice in the Professions: Women Doctors and Lawyers in Third Republic France». Journal of Women's History, 19 (3), 60-86.

FLOOD, John (1991). "Doing business: The management of uncertainty in lawyers' work». Law and Society Review, 25 (1), 41-71.

- (2011). «The re-landscaping of the legal profession: Large law firms and professional re-regulation». Current Sociology, 59, 507-529.

Guillén, Mauro (1990). «Profesionales y burocracia: Desprofesionalización, proletarización y poder profesional en las organizaciones complejas». Revista Española de Investigaciones Sociológicas, 51, 35-51.

GuTIÉRreZ, Carme; LOPE, Andreu y TORNS, Teresa (2002). L'advocacia: una qüestió de gènere?: L'exercici profesional de les advocades de Barcelona. Bellaterra: Servei de Publicacions. Universitat Autònoma de Barcelona.

Heinz, John P.; Nelson, Robert L. y Laumann, Edward O. (2001). "The Scale of Justice: Observations on the transformation or Urban Law Practice». Annual Review of Sociology, 27, 337-362.

InSTITUTO DE INVESTIGACIONES Y ANÁLISIS SOCIO-JuRÍDICOS (2003). La imagen de la abogacía en la sociedad española: Primer barómetro externo de opinión del Consejo General de la Abogacía. Madrid: Consejo General de la Abogacía Española.

INSTITUTO DYM (2003). Abogados y nuevas tecnologías (informe técnico no publicado). Madrid: Instituto DYM.

Instituto NaCiONAL DE EsTAdística (2012). Estadistica de la Enseñanza Universitaria en España. Curso 2010-2011. Licenciaturas [en línea]. Madrid: INE. <http://www. ine.es/inebmenu/mnu_educa.htm> [Consulta: 8 agosto 2012].

KaY, Fiona M. y HaGAN, John (1998). «Raising the Bar: The gender stratification of Law-firm capital». American Sociological Review, 63, 728-743.

Metroscopia (2005). La abogacía vista por los abogados: Segundo barómetro externo de opinión del Consejo General de la Abogacía Española. Madrid: Consejo General de la Abogacía Española.

- (2008). La abogacía vista por los abogados: Tercer barómetro interno de opinión del $C G A E$. Madrid: Consejo General de la Abogacía Española.

- (2011). La imagen de los abogados y de la justicia en la sociedad española: Cuarto barómetro externo de opinión. Madrid: Consejo General de la Abogacía Española.

Mullerat, Ramón (2001). «La profesión de abogado en 2010». La Ley: Revista Jurídica Española de Doctrina, Jurisprudencia y Legislación, 1, 1802-1806.

ORDRE DES AVOCATS À LA COUR DE PARIS (2002). Usage et Image des avocats auprès de la cible des particuliers (informe técnico no publicado). París: Ordre des Avocats à la Cour de Paris.

Pérez Moreno, José Arturo (2003). El ejercicio de la abogacía [en línea]. Ponencia presentada en el VIII Congreso de la Abogacía Española. Salamanca. <http://www. cgae.es/especial/index.htm> [Consulta: 27 julio 2011].

PESTANA, José Vicente (2008). «Professionals de l'Advocacia de l'ICAB. Universitats de procedència i estudis de formació contínua a la seu col-legial». En: JuANí RAMON, Noemí (dir.). Informe 2008 sobre la Justícia, Il-lustre Col.legi d'Advocats de Barcelona. Barcelona: Il.lustre Col.legi d'Advocats de Barcelona. 
POWELL, Michael J. (1985). «Developments in the regulation of lawyers: Competing segments and market, client, and government controls». Social Forces, 64 (2), 281-305.

Rodríguez Ávila, Nuria (2000). «Mercado y formas de trabajo: Los abogados ante el siglo XX. El caso catalán». Hitos de Ciencias Económico Administrativas, 6 (16), 5-18.

- (2001). Los abogados ante el siglo XXI [en línea]. Tesis doctoral. Barcelona: Universitat de Barcelona. <http://tdx.cat/bitstream/handle/10803/2875/NRA_TESIS. pdf?sequence $=1>$ [Consulta: 27 julio 2011].

- (2008). Manual de sociología de las profesiones. Barcelona: Publicacions i Edicions de la Universitat de Barcelona.

RoJAS, Mauricio (2009). "No el abogado, "mejor el doctor": La imagen social del profesional en Derecho». Revista Estudios Socio-Jurídicos, 11 (2), 291-298.

SÁEZ, Juan; SÁNCHEZ, Mariano y SÁNCHEZ, Elena (2009). "¿Sociología de las profesiones en España?: Entre la carencia y la necesidad de consolidación». Universitas Tarraconensis: Revista de Ciències de l'Educació, XXXIII (1), 15-101.

SÁnCHEZ, Mariano y SÁEZ, Juan (2009). «El estudio de las profesiones: La potencialidad del concepto de profesionalización». Universitas Tarraconensis: Revista de Ciències de l'Educació, XXXIII (1), 103-117.

Soriano Díaz, Ramón; RuIz de la Cuesta, Antonio y GonZÁlez-Tablas, Rafael (1987). Los abogados andaluces: análisis sociológico. Sevilla. Instituto de Desarrollo Regional.

StumpF, Harry P.; Schroerluke, Henry P. y Dill, Forrest D. (1971). «The legal profession and legal services: Explorations in local Bar politics». Law and Society Review, 6 (1), 47-67.

TOHARIA, Juan José (1984). «Los españoles ante el juicio con jurado». Revista Española de Investigaciones Sociológicas, 25, 251-263.

Troiano, Helena (2005). «Consistencia y orientación del perfil profesional, percepción del mercado laboral y reacciones previstas ante posibles dificultades de inserción». Papers, 76, 167-197.

Wallace, Jean E. (1997). «It's about time: A study of hours worked and work spillover among Law Firm Lawyers». Journal of Vocational Behavior, 50, 227-248.

- (1999). "Work-to-nonwork conflict among married male and female lawyers». Journal of Organizational Behavior, 50, 797-816.

ZARAGOZA, Ángel (1975). «Los despachos colectivos y la empresa legal como modos de ejercer la abogacía». Sociología y Psicología Jurídicas, II, 63-74. 